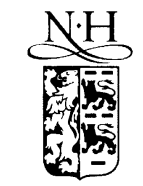

ELSEVIER
Available online at www.sciencedirect.com

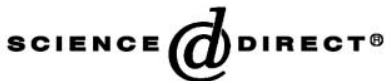

Nuclear Instruments and Methods in Physics Research B 200 (2003) 363-370

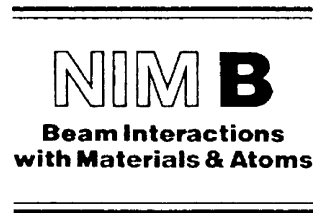

www.elsevier.com/locate/nimb

\title{
The structure of organic langmuir films on liquid metal surfaces
}

\author{
H. Kraack ${ }^{\text {a }}$, M. Deutsch ${ }^{\text {a,* }}$, B.M. Ocko ${ }^{b}$, P.S. Pershan ${ }^{\text {c }}$ \\ a Physics Department, Bar Ilan University, Ramat Gan 52900, Israel \\ b Physics Department, Brookhaven National Laboratory, Upton, NY 11973, USA \\ ${ }^{\mathrm{c}}$ Physics Department and Division of Applied Sciences, Harvard University, Cambridge, MA 02138, USA
}

\begin{abstract}
Langmuir films (LFs) on water have long been studied for their interest for basic science and their numerous applications in chemistry, physics, materials science and biology. We present here $\AA$-resolution synchrotron X-ray studies of the structure of stearic acid LFs on a liquid mercury surface. At low coverage, $\geqslant 110 \AA^{2} / \mathrm{mol}$, a $2 \mathrm{D}$ gas phase of flat-lying molecules is observed. At high coverage, $\leqslant 23 \AA^{2} / \mathrm{mol}$, two different hexatic phases of standing-up molecules are observed. At intermediate coverage, $52 \leqslant A \leqslant 110 \AA^{2} / \mathrm{mol}$, novel single- and double-layered phases of flat-lying molecular dimers are found, exhibiting a 1D in-layer order. Such flat-lying phases were not hitherto observed in any LF. Measurements on LFs of fatty acids of other chain lengths indicate that this structure is generic to chain molecules on mercury, although the existence of some of the flat-lying phases, and the observed phase sequence, depend on the chain length. Organic LFs on $\mathrm{Hg}$, and in particular the new flat-lying phases, should provide a broader nano-structural tunability range for molecular electronic device construction than most solid-supported self-assembled monolayers used at present.

(c) 2002 Elsevier Science B.V. All rights reserved.
\end{abstract}

Keywords: Langmuir films; Monolayers; Surface; Liquid metals; 2D phases

\section{Introduction}

The advent of widely accessible, dedicated synchrotron radiation sources in the early 1980s revolutionized the field of structural studies of surfaces by X-ray methods. In particular, novel synchrotron-based X-ray reflectivity and grazing incidence diffraction methods were developed to study the surfaces of liquids [1,2]. The first nanoscale measurement of the surface structure of

\footnotetext{
${ }^{*}$ Corresponding author. Tel.: +972-351-84765; fax: +9723535-3298.

E-mail address: deutsch@mail.biu.ac.il (M. Deutsch).
}

water [3] paved the way to the $\AA$-resolution determination of the structure of Langmuir films (LFs) on water [4]. These films have been investigated by macroscopic methods such as surface tension, surface potential, etc. for over a century $[5,6]$, due to their fundamental importance in many branches of basic and applied science. They have been studied as models for 2D matter in physics, for the cell membrane in biology, as preferred-orientation crystal nucleators in chemistry, and as a route to nano-engineering and molecular electronics in materials science [6,7]. Since the first in situ X-ray studies of LFs in 1987 [4], many such studies [6] revealed a wealth of structures, phases and transitions in chain molecules on various 
aqueous subphases. In particular, their (length, area, temperature) phase diagram has been determined in detail, showing a number of hexatic, 1Dordered and 2D-ordered phases [6,8]. However, in all these studies the strong hydrophobic repulsion between the chains and the subphase dominated the molecular alignment, orienting the long axis of the molecules at, or close to the surface normal. We report here a study of the coverage dependence of the structure of stearic acid LFs on a liquid mercury subphase, using pressure/area isotherm measurements and Angström-resolution X-ray techniques. The high-coverage phases are found to be similar to those observed on aqueous subphases [4]. The low-coverage phases, however, consist of single and double layers of flat-lying molecular dimers, the X-ray diffraction patterns of which suggest a 2D-smectic-like order. A similar study of several other fatty acids of different chain lengths demonstrates that these phases are universal, although the number of flat-lying layers, and thus the phase sequence observed upon varying the coverage, changes systematically with chain length. Preliminary measurements indicate that the nature of the head group (methylene, alcohol, acid, thiol, etc.) plays an important role in determining the number and types of the flat-lying phases, and the existence of the high-coverage phases of surfacenormal molecules.

Although there is a wealth of thermodynamic data available for organics on mercury [9], only three structural X-ray studies [10-12] were published prior to the study reported here. None of these determined the variation of the LFs' structure with coverage. Moreover, all of them employed only a single high-coverage LF, and only close-packed phases with surface-normal molecules were found. For a typical fatty acid, like the stearic acid $\left(\mathrm{CH}_{3}-\left(\mathrm{CH}_{2}\right)_{16}-\mathrm{COOH}\right)$, the estimated carboxylic headgroup-Hg subphase bond strength is $\sim 1.5 \mathrm{~kJ} / \mathrm{mol}$ [10]. This is well below the carboxyl-water hydrogen bond strength, $\leqslant 10 \mathrm{~kJ} / \mathrm{mol}$ [13]. Stearic, and other fatty acids molecules that form LFs on mercury are expected to have a high surface mobility thereby allowing the formation of thermal equilibrium structures at all coverages. This expectation is confirmed in this study by $\mathrm{X}$ ray reflectivity (XR) [14], grazing incidence dif- fraction (GID) [14,15], Bragg rod (BR) [14,15] and surface-pressure $(\pi)$ /molecular-area $(A)$ isotherm $[9,16]$ measurements, as detailed below.

\section{Experimental}

The surface tension [16] and X-ray [14,15] methods employed were discussed extensively in the literature and are, therefore, reviewed here only briefly.

A home-made Langmuir trough of area $6.5 \times$ $17.5 \mathrm{~cm}^{2}$ was employed in the measurements. The trough was enclosed in a hermetically sealed box, equipped with a film balance to measure the surface pressure $\pi$. The balance used an $\mathrm{Hg}$-amalgamated platinum Wilhelmi plate. The molecular area $A$ was varied by stepwise deposition of metered volumes of the chloroform-dissolved sample. Samples, with purities $>99 \%$, were purchased from Aldrich/Fluka/Sigma and used without further purification.

For the X-ray measurements the Kapton windows were used, and the box assembly was mounted on the sample stage of a liquid diffractometer. During the X-ray measurements the box was filled with helium to minimize X-ray damage and parasitic diffuse scattering. Measurements were carried out at the Harvard/Brookhaven liquid surface diffractometer, beamline X22B, NSLS, at a wavelength of $1.556 \mathrm{~A}$. The XR measurements employed a point detector, while the GID measurements used a $100 \mathrm{~mm}$ linear position sensitive detector allowing a simultaneous measurement of a full Bragg rod. The XR data analysis employed computer fits to standard few-layer box models [14,17].

\section{Results}

We now discuss results obtained for fatty acids, focusing in detail on stearic acid LFs.

\subsection{Stearic acid}

\subsubsection{Pressurelarea isotherm}

The $\pi / A$ isotherm we measured for stearic acid on $\mathrm{Hg}$ is shown in Fig. 1, along with the previously 
measured [16] isotherm on water. The most outstanding feature in Fig. 1 is the much larger range of both $A$ and $\pi$ accessible on $\mathrm{Hg}$ as compared with water. The fast-increasing region at $110 \leqslant A \leqslant 200$ $\AA^{2} / \mathrm{mol}$ is very well fitted by the $2 \mathrm{D}$ Vollmer equation (dashed line), $\pi\left(A-A_{0}\right)=k_{\mathrm{B}} T$, where $k_{\mathrm{B}}$ is the Boltzmann constant and $A_{0}$ is the exclusion area due to the finite molecular size [9]. The fitted value $A_{0}=(112 \pm 10) \AA^{2} / \mathrm{mol}$ coincides with the calculated area of a flat-lying molecule: 24.4 $\AA \times 4.85 \AA \approx 118 \AA^{2}$, using well known values for the length and the nearest-neighbour chain spacing [18]. This leads to the immediate conclusion that for $A \geqslant 110 \AA^{2} / \mathrm{mol}$ the LF is a gas of flat-lying molecules, and that at point (2) a closely packed single layer of such molecules is obtained. These conclusions are strongly supported by the X-ray measurements detailed below. Upon decreasing $A$ from $110 \AA^{2} / \mathrm{mol}$ several additional features are observed in the isotherm: kinks, slow- and fastrising $\pi$ regions, and a plateau. However, based on the isotherm alone, no definite structural meaning can be assigned to these features. The X-ray measurements discussed below reveal clearly the structure of the LF in the regions marked by these features.

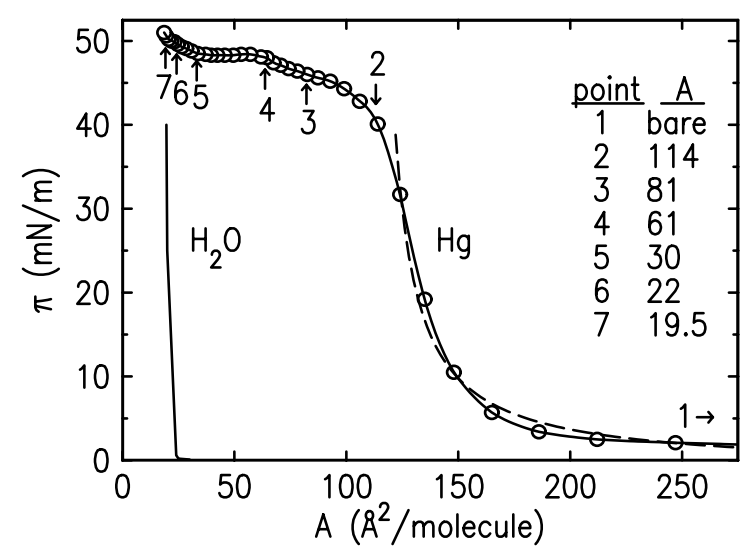

Fig. 1. Surface pressure $(\pi) /$ molecular area $(A)$ isotherms of a LF of stearic acid on mercury (circles + solid line), and on water (solid line) at room temperature. The numbers mark the points where XR curves were measured. A fit to the Vollmer equation, the isotherm of a hard-sphere gas, (dashed line) yields a $112 \AA^{2}$ molecular-size exclusion area, coinciding with that of a flatlying molecule.
3.1.2. X-ray reflectivity: the surface-normal structure

Fig. 2(a) presents some of the measured Fresnel-normalized XR curves (circles) taken at the points marked on Fig. 1, along with model fits (lines). The corresponding electron density profiles are shown in Fig. 2(b). The fit at (2) shows a uniform LF of thickness $d=4.8 \AA$ and electron density $\rho=0.30 \mathrm{e} / \AA^{3}$. These values agree closely with the inter-chain distance $[17,18]$ and the electron density [17,19] of close-packed alkyl chains. This and the isotherm strongly support the conclusion that for $A \geqslant 110 \AA^{2} / \mathrm{mol}$ the LF consists of a single layer of flat-lying molecules. Note that the observation of such thin films, $d \leqslant 5 \AA$ by XR is possible only on liquid metal surfaces, where the high surface tension, $\gamma \approx 500 \mathrm{mN} / \mathrm{m}$ for $\mathrm{Hg}$, results in a very low surface roughness, $\sigma \leqslant 1 \AA$. The larger surface roughness $\sigma \approx 2.7 \AA$ of water [3], where $\gamma \approx 72 \mathrm{mN} / \mathrm{m}$, limits reliable XR studies to thickness values $d \geqslant 12-15 \AA$.

The thickness of $d=9.6 \AA$ obtained from the fit of the XR curve at (4), $A=61 \AA^{2} / \mathrm{mol}$, reveals that

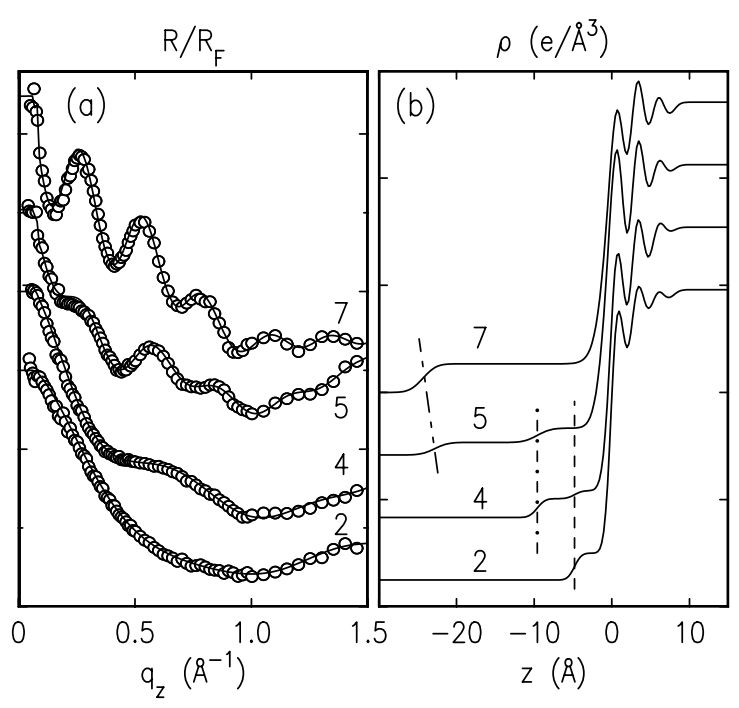

Fig. 2. (a) The measured and Fresnel-normalized $X R, R / R_{F}$ (circles) and model fits (lines) for the points numbered on Fig. 1. (b) The surface-normal electron density profiles, $\rho(z)$, derived from the fits. The $\mathrm{Hg}$ surface is at $z=0$. The dash, dash-dot and dash-dot-dot lines denote the tops of the flat-lying single and double layers, and the standing-up monolayer, respectively. Curves are shifted for clarity. 
a second flat-lying layer is formed at the surface on top of the first one in the increasing- $\pi$ part of the isotherm, $110 \geqslant A \geqslant 60 \AA^{2} / \mathrm{mol}$. The fit at (4) yields $100 \%$ coverage for the first layer, and a density equivalent to $75 \%$ coverage for the second layer, in agreement with the amount of material deposited. The XR results show that $100 \%$ coverage is reached for the second layer at $A \approx 52 \AA^{2} /$ mol. This is close to the kink in the isotherm marking the onset of the plateau at $A=57 \AA^{2} / \mathrm{mol}$, and to $118 / 2 \approx 59 \AA^{2} / \mathrm{mol}$, the calculated area for a two-layered film based on the molecular dimensions. This strongly supports the present interpretation of a double-layer phase in this $A$ range over the previous interpretation, based on the isotherm alone, of a single molecular layer consisting of surface-parallel molecules part of which are strongly bent in the surface-normal direction. To explain the continuous change in area with coverage it was postulated that the position of the bend along the chain changed with the area $A$ [9]. The existence of the single- and double-layers of flat-lying molecules for $A \geqslant 52 \AA^{2} / \mathrm{mol}$ indicates that the structure is dominated by the strong chain-subphase attraction, which induces a surface-parallel orientation of the molecules of the LF. This is in contrast with LFs on water where the strong hydrophobic repulsion induces a surface-normal molecular orientation at all coverages. The attraction to the $\mathrm{Hg}$ surface here is insufficient to induce a third layer of flat-lying molecules. When $A$ is decreased below $52 \AA^{2} / \mathrm{mol}$ the molecules start orienting roughly normal to the surface thus decreasing the average area per molecule on the surface.

The XR reflectivity curve (5) at $A=30 \AA^{2} / \mathrm{mol}$, typical of the plateau region of the isotherm, could be fitted only by assuming a two-phase coexistence between a double layer of flat-lying molecules and a monolayer of standing-up molecules. The fits, constrained by keeping the double-layer's $d$ and $\rho$ fixed at the $A=52 \AA^{2} / \mathrm{mol}$ values, show for (5) that $47 \%$ of the surface area is covered by the monolayer, which includes $69 \%$ of the molecules. The fitted thickness of the standing-up monolayer, $d=22.3 \AA$, is less than the extended molecular length, $24.4 \AA$. This can be interpreted as being due to a $\sim 24^{\circ}$ tilt of the molecules from the surface normal. This interpretation is shown to be correct by the GID results. Decreasing $A$ from 35 to $25 \AA^{2} /$ mol increases the monolayer-covered area from $24 \%$ to $77 \%$, with $d$ increasing from 22.1 to $23.2 \AA$. This is accompanied by a decrease in the molecular tilt from $\sim 25^{\circ}$ to $\sim 18^{\circ}$.

The coexistence range ends at $A \approx 23 \AA^{2} / \mathrm{mol}$. For lower $A$ the XR can only be fitted assuming a single monolayer of standing-up molecules, with the carboxylic heads residing at the $\mathrm{Hg}$ surface. Curve (7), $A=19.5 \AA^{2} / \mathrm{mol}$, is typical of this region, with a layer thickness of $24.3 \AA$ and a tilt $\leqslant 5^{\circ}$ (the XR detectible limit). The XR-derived structure in this range is very similar to that of LFs of stearic acid on water at similar $A$ s $[6,8]$. This demonstrates the dominance of chain-chain interactions over chain-subphase attraction in this region.

\subsubsection{Grazing incidence diffraction: the surface- parallel structure}

Grazing incidence diffraction measurements show a fundamentally different in-plane structure for the flat-lying and standing-up phases. Both low coverage phases, the single- and double-layers of flat-lying molecules, show several orders of low- $q_{\|}$ GID peaks, all multiples of $q_{\|}=0.120 \AA^{-1}$, as shown in Fig. 3. This indicates a well-ordered structure with a repeat distance $d_{\|}=52.4 \AA$, which is $6-8 \%$ larger than the layer spacing $d_{\text {bulk }}=49.3 \AA$ of bulk stearic acid [20], and twice the extended molecule's length, 48.8 A. All crystalline polymorphs of stearic acid consist of dimers, hydrogen bonded by the carboxylic headgroups of the two molecules [18]. The close correspondence between $d_{\|}$and $d_{\text {bulk }}$ implies that our low-coverage phases consist of in-plane ordered uniform-width stripes of flat-lying dimers, oriented along the stripe's width. The BR results, discussed elsewhere [21], are consistent with this conclusion. The repeat distances of metal stearate Langmuir-Blodgett multilayers were found to range from $49.9 \AA$ ( $\mathrm{Mn}$ stearate [22]) to $50.6 \AA$ (Pb stearate [23]), all within $\sim 1 \AA$ of $d_{\text {bulk }}$, and significantly smaller than $d_{\|}=52.4 \AA$ of our flat-lying phases. Thus, repeat distance considerations can neither support nor rule out a possible incorporation of $\mathrm{Hg}$ atoms into the flat-lying dimer's center, as found in self- 


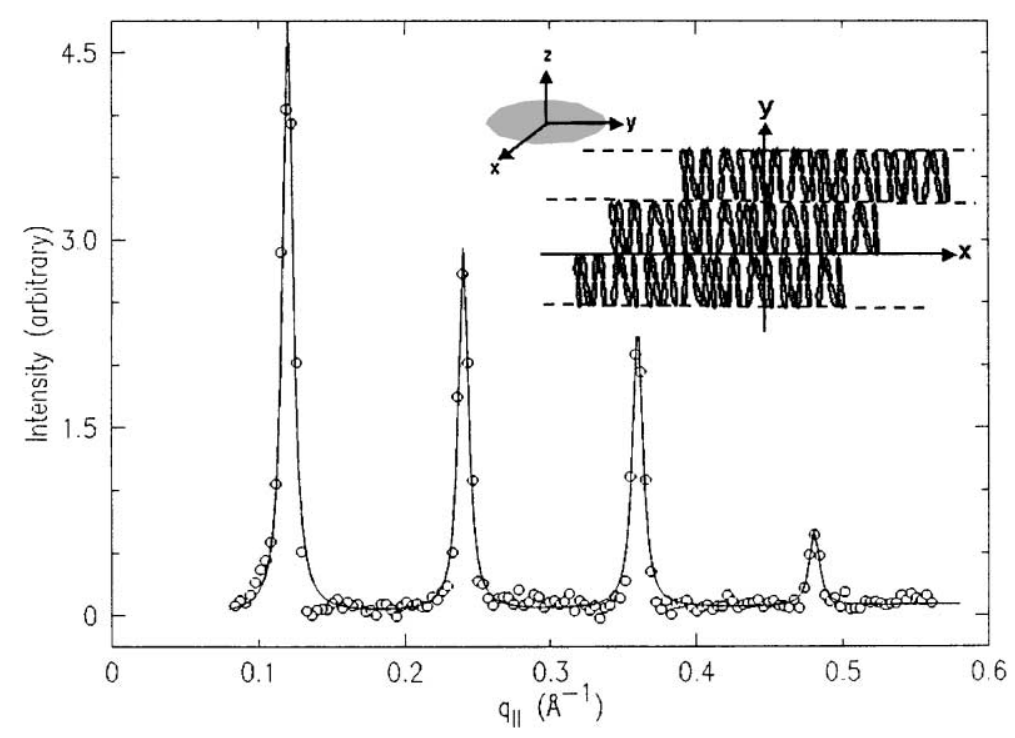

Fig. 3. Low- $q$ grazing incidence diffraction of the flat-lying double-layer phase of stearic acid on mercury. The four orders of the diffraction peak show a repeat distance corresponding to the length of a molecular dimer. The absence of high- $q$ peaks, which would correspond to order normal to the dimer's long axis indicate a 1D smectic-like order in the quasi-2D layer, as shown in the cartoon.

assembled multilayers of surface-normal thiol molecules [24]. The origin of the $\sim 3 \AA$ difference between the repeat distances of flat-lying (here) and surface-normal (bulk) dimer phases is unclear and merits further study.

It is important to note that no GID peaks were found for the flat-lying phases at $1.2 \leqslant q_{\|} \leqslant 1.4 \AA^{-1}$. Peaks in this range would correspond to the existence of order in the packing of the chains in the inplane direction normal to the flat-lying dimer's long axis. Since we did not observe such peaks we conclude that only short range order may exist in this direction. Thus, long range order exists in the flatlying phases only along the long axis of the dimers, but not perpendicular to it. The proposed structure is shown in the inset in Fig. 3, and suggest a 2D smectic-like structure for the flat-lying phases, i.e. a $1 \mathrm{D}$ order within a quasi-2D layer.

The high-coverage standing-up phases show measurable GID peaks for $A \leqslant 35 \AA^{2} /$ mol only. At the high-coverage end of this range, a NNN molecular tilt and a distorted hexagonal in-plane packing are observed [6,21]. As coverage is increased, the tilt reduces continuously, reaching $0^{\circ}$ tilt, and an undistorted hexagonal in-plane packing, at $\sim 20 \AA^{2} / \mathrm{mol}$. Two distinct hexatic phases are clearly identified within this range [21]: an Overbeck-Möbius $\left(\mathrm{O}_{\mathrm{v}}\right)$ phase $[6,25]$ at the high- $A$ end, and a LS phase [6] at the low- $A$ end. The structural parameters of all phases, derived from the GID and reflectivity measurements, are listed in Table 1, along with their uncertainties. The same $\mathrm{O}_{\mathrm{v}}$ and LS phases were also found, with very similar unit cell dimensions and tilts, for stearic acid LM on water at high $\pi$ values [8]. The lowersymmetry phases found on water [6], some exhibiting NN tilts and almost all showing herringbone order for the molecular plane, are not detected here. This is not surprising considering that the surface pressures at which these phases occur in water-supported LFs are much lower than the $\pi \geqslant 48 \mathrm{mN} / \mathrm{m}$ at which the standing-up phases are observed in our Hg-supported LFs.

\subsection{Other fatty acids}

The isotherms measured for several fatty acids (denoted $\mathrm{C}_{n} \mathrm{OOH}$ ) with different number of carbons, $n$, on $\mathrm{Hg}$ at room temperature are shown in Fig. 4. An inter-comparison of the isotherms reveals some clear trends in the variation of the various features with chain length. 
Table 1

Structural parameters for stearic acid LFs on mercury at different coverages A and the corresponding phases

\begin{tabular}{|c|c|c|c|c|c|c|c|c|c|c|}
\hline $\begin{array}{l}A \\
\left(\AA^{2} / \mathrm{mol}\right)\end{array}$ & Phase & $\begin{array}{l}d \\
(\AA)\end{array}$ & $\begin{array}{l}\sigma \\
(\AA)\end{array}$ & $\begin{array}{l}q_{\|, p}(11) \\
\left(\AA^{-1}\right)\end{array}$ & $\begin{array}{l}q_{\|, p}(02) \\
\left(\AA^{-1}\right)\end{array}$ & $\begin{array}{l}q_{z, p}(02) \\
\left(\AA^{-1}\right)\end{array}$ & $\begin{array}{l}q_{z, p}(11) \\
\left(\AA^{-1}\right)\end{array}$ & $\begin{array}{l}a \\
(\AA)\end{array}$ & $\begin{array}{l}b \\
(\AA)\end{array}$ & $\begin{array}{l}\theta \\
\left({ }^{\circ}\right)\end{array}$ \\
\hline 114 & SL & 4.8 & 0.7 & & & & & & & \\
\hline 61 & SL/DL & $4.8 / 9.6$ & 0.9 & & & & & & & \\
\hline 52 & DL & 9.6 & 1.3 & & & & & & & \\
\hline 30 & $\mathrm{DL} / \mathrm{O}_{\mathrm{v}}$ & $9.6 / 22.3$ & 1.4 & 1.377 & 1.451 & 0.78 & 0.39 & 4.92 & 9.13 & 30 \\
\hline 22 & $\mathrm{O}_{\mathrm{v}}$ & 23.4 & 1.1 & 1.459 & 1.487 & 0.50 & 0.27 & 4.85 & 8.61 & 19 \\
\hline 19.5 & LS & 24.3 & 1.4 & 1.525 & 1.525 & 0 & 0 & 4.76 & 8.24 & 0 \\
\hline \multicolumn{2}{|c|}{ Uncertainty } & \pm 0.6 & \pm 0.2 & \pm 0.003 & \pm 0.003 & \pm 0.03 & \pm 0.03 & \pm 0.01 & \pm 0.01 & \pm 3 \\
\hline
\end{tabular}

$\mathrm{DL}, \mathrm{SL}, \mathrm{O}_{\mathrm{v}}$ and LS denote the flat-lying double and single layers, and the standing-up Overbeck and hexagonal phases, respectively. $d$ and $\sigma$ are the layer thickness and roughness. $q_{\|, p}$ and $q_{z, p}$ are the GID and BR peak positions for the standing-up phases, $\theta$ is the molecular tilt, and $a$ and $b$ are the dimensions of the rectangular unit cell.

Note first the linear variation with chain length of the position of the steep rise at large $A$. The Vollmer equation, discussed above, agrees well with all isotherms in this region. The $A_{0}$ values obtained from the fits are in all cases in excellent agreement with the area of a flat-lying molecule, calculated from $A=l \times 4.8 \AA^{2}$, where $l$ is the length of the molecule, as shown in the inset to Fig. 4. This supports further the conclusions drawn above that the large- $A$ region of the isotherm is that of a 2D "gas" of flat lying molecules, and the first horizontal bend following the steep

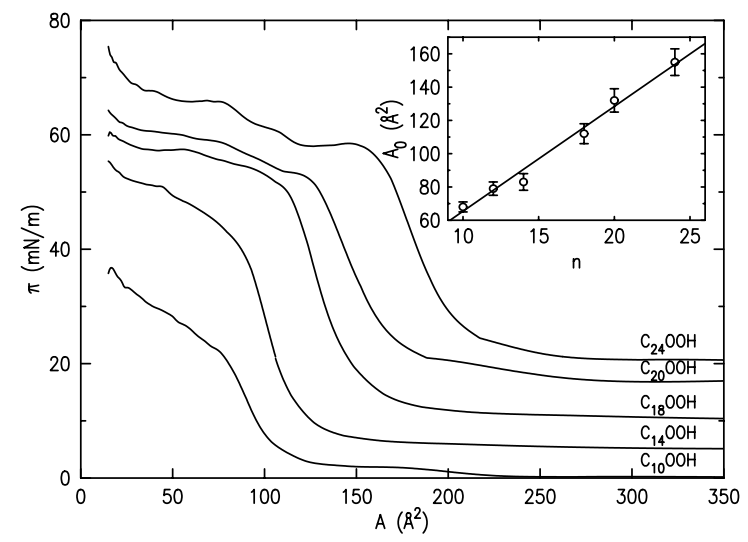

Fig. 4. Isotherms of fatty acid LFs (of carbon numbers $10 \leqslant n \leqslant 24$ ) on mercury at room temperature. Inset: the molecular exclusion areas $A_{0}$ (circles) obtained from fits of the $2 \mathrm{D}$ "ideal" gas formula to the steeply-rising part of the isotherms and the corresponding calculated areas of the flat-lying molecules (line). rise marks the completion of a close-packed single layer of flat-lying molecules on the $\mathrm{Hg}$ surface.

For all $n$ measured $(n=10,12,14,18,20,24)$ the flat-lying single layer phase is observed just below the steep increase at $A=A_{0}$. The fully standing-up phase(s) at the low- $A$ limit are also observed. However, at this point no X-ray data is available to determine the structure of these phases. The qualitative shape and the magnitudes of the surface pressure at the points that the single and double flat-lying layers achieve complete coverage are the same for all the fatty acids with $12 \leqslant n \leqslant 24$. The same is true for the flat part of the isotherms at half the fully compressed single-layer's area $/ \mathrm{mol}$. This indicates the same sequence of phases for all chain lengths studied: first flat-lying single- and double-layers followed by one or more standing-up phases. Preliminary $\mathrm{X}$-ray data show clearly that the double-layer phase of flat-lying molecules also appears at least for $\mathrm{C}_{24} \mathrm{OOH}$. The absence of a clear plateau in the isotherm of $\mathrm{C}_{10} \mathrm{OOH}$ and the much lower surface pressure at the first "bend" $\left(\sim 70 \AA^{2} / \mathrm{mol}\right)$ suggests that the decanoic acid $\mathrm{C}_{10} \mathrm{OOH}$ only exhibits a single layer, and no double layer. We attribute the lack of a double-layer for $\mathrm{C}_{10} \mathrm{OOH}$ to a larger degree of disorder for this molecule, the bulk melting point of which is just slightly above room temperature, $T_{\mathrm{m}}=31.6{ }^{\circ} \mathrm{C}$. Further $\mathrm{X}$-ray measurements, now in progress, are expected to elucidate the details of the structure and phase sequences of each of these LFs and the $n$ dependence of the phase diagram. 


\section{Conclusions}

The results presented above highlight the importance of the surface-chain interaction for the determination of the structure and phase sequence of LFs on both mercury and water. The attractive interaction in our case induces flatlying single- and double-layer phases. By imposing a high $\pi$-threshold on the standing-up phases, this attraction is also responsible for the absence of the low- $\pi$ standing-up phases observed in water-supported LFs. The confinement of the LF to the surface by the strong chain-mercury attraction allows achieving much higher surface pressures without causing the LF to buckle and collapse, as compared to aqueous subphases. For stearic acid novel 2D-smectic-like phases are observed, whose structural unit is a dimer of flat-lying molecules connected at their carboxylic headgroups. The possibility that the headgroup region contains also one or more mercury atoms cannot be ruled out. At high coverages, phases of standing-up molecules are observed, similar to the hexatic Ov and LS phases found for watersupported stearic acid LFs. A coexistence range between flat-lying and standing-up phases is also found. First measurements on other fatty acids indicate a similar phase sequence for chain lengths $10 \leqslant n \leqslant 24$ at room temperature, though the maximal number of layers of flat-lying molecules varies with $n$. Measurements in progress on related molecules like alkanes, alcohols and thiols indicate that the headgroup's polarity plays an important role in determining the transition to the standing-up phases and, indeed, their very existence for a given molecule [26]. The ability to tune the structure of $\mathrm{Hg}$-supported LFs over much larger coverage and surfacepressure ranges than those of water-supported LFs, and in particular the new flat-lying phases, should prove very advantageous for the construction and study of nano-sized electronic devices [7,27], and may help resolving fundamental controversies such as whether conformational disorder inhibits or promotes charge transfer across a monolayer in $\mathrm{Hg}$-organics heterostructured devices [28].

\section{Acknowledgements}

Support to P.S.P. by the US DOE (grant no. DE-FG02-88-ER45379) and the National Science Foundation (grant no. DMR-01-124936) and to M.D. by the US-Israel Binational Science Foundation, Jerusalem, is gratefully acknowledged. We thank D. Vaknin (Ames) for generous advice and drawings of the trough. BNL is supported by the US DOE (contract no. DE-AC02-98CH10886).

\section{References}

[1] J. Als-Nielsen, F. Christensen, P.S. Pershan, Phys. Rev. Lett. 48 (1982) 1107;

P.S. Pershan, J. Als-Nielsen, Phys. Rev. Lett. 52 (1984) 759.

[2] J. Penfold, Rep. Prog. Phys. 7 (2001) 777.

[3] A. Braslau et al., Phys. Rev. Lett. 54 (1985) 114.

[4] S. Grayer Wolf et al., Nature 328 (1987) 63;

K. Kjaer et al., Phys. Rev. Lett. 58 (1987) 2224;

P. Dutta et al., Phys. Rev. Lett. 58 (1987) 2228.

[5] A. Pockels, Nature 43 (1891) 437.

[6] V.M. Kaganer et al., Rev. Mod. Phys. 71 (1999) 779; I. Kuzmenko et al., Chem. Rev. 101 (2001) 1659; R.H. Tregold, Order in Thin Organic Films, Cambridge University, Cambridge, 1994.

[7] J. Jortner, M. Ratner (Eds.), Molecular Electronics, Blackwell, Oxford, 1997.

[8] I.R. Peterson et al., J. Phys. Chem. B 102 (1998) 9437.

[9] T. Smith, Adv. Coll. Interface Sci. 3 (1972) 161.

[10] S.W. Barton et al., Langmuir 4 (1988) 233.

[11] B. Harzallah et al., J. Chim. Phys. (France) 93 (1996) 1202.

[12] O.M. Magnussen et al., Nature 384 (1996) 250.

[13] R.C. Weast, M.J. Astle, W.H. Beyer (Eds.), Handbook of Chemistry and Physics, 66th ed., CRC, Boca Raton, 1986, p. F174.

[14] M. Deutsch, B.M. Ocko, in: G.L. Trigg (Ed.), Encyclopedia of Applied Physics, Vol. 23, VCH, New York, 1998, p. 479; J. Daillant, A. Gibaud (Eds.), X-Ray and Neutron Reflectivity: Principles and Applications, Springer, Berlin, 1999.

[15] J. Als-Nielsen et al., Phys. Rep. 246 (1994) 252; J. Daillant, M. Alba, Rep. Prog. Phys. 63 (2000) 1725.

[16] G.L. Gaines, Insoluble Monolayers at Liquid-Gas Interfaces, Wiley, New York, 1966.

[17] B.M. Ocko et al., Phys. Rev. E 55 (1997) 3164.

[18] D.M. Small, The Physical Chemistry of Lipids, Plenum, New York, 1986.

[19] X.Z. Wu et al., Phys. Rev. Lett. 70 (1993) 958.

[20] M. Popescu et al., Thin Solid Films 304 (1997) 323.

[21] H. Kraack et al., Science 298 (2002) 1404. 
[22] Hazra et al., Physica B 283 (2000) 45.

[23] P. Ganguly et al., J. Phys. Chem. 97 (1993) 11965.

[24] M. Deutsch et al., in: A. Ulman (Ed.), Self-Assembled Monolayers of Thiols, Academic, San Diego, 1998.

[25] G.A. Overbeck, D. Möbius, J. Phys. Chem. 97 (1993) 7999.

[26] H. Kraack et al., unpublished.
[27] M.A. Rampi et al., Appl. Phys. Lett. 72 (1998) 1781;

K. Slowinski et al., J. Am. Chem. Soc. 121 (1999) 7257.

[28] C. Boulas et al., Phys. Rev. Lett. 76 (1996) 4797;

A. Haran et al., Science 263 (1994) 948;

K. Slowinski, M. Majda, J. Electroanal. Chem. 491 (2000) 139. 